\title{
Induction of Anti-Proliferative and Apoptotic Effects of Sorafenib Using miR-27a Inhibitor in Hepatocellular Carcinoma Cell Lines
}

\author{
Abdel Hady A Abdel Wahab ${ }^{1 *}$, Eman G Ayad ${ }^{2}$, Mohga S Abdulla², Hayat M \\ Sharada $^{2}$, Abeer M Ashmawy ${ }^{1}$
}

\begin{abstract}
Objective: The purpose of the current study was to investigate the possible anti-tumor effect of miR-27a inhibitor in combination with Sorafenib (SOR) on cell proliferation and apoptosis of hepatocellular carcinoma cell lines. Methods: Transient transfection by oligo-miR27a inhibitor (miR-27ai) was used in this study for targeting the oncogenic miR-27a in HepG2 and Huh7 cells followed by SOR treatment. Cell viability was measured using SRB assay. The cell cycle and apoptosis were assessed by flow cytometry assay. Moreover, the level of oncogenic miR-27a was evaluated in 19 tissues of primary HCC patients as well as cell lines using qRT-PCR assay. Finally, caspase-3 activity was determined using ELISA assay. Results: Significant up-regulation of miR-27a expression was reported in HCC patients confirming its oncogenic role. Treatment of cells with SOR following transfection with miR-27ai declined cell viability significantly compared with either control or single agent treatment $(\mathrm{p} \leq 0.05)$. Highly significant decreasing in the number of cell in S-phase associated with increasing in G0-phase was also observed. Furthermore, apoptotic rate was highly significantly increased for transfected/SOR treated cells $(\mathrm{p} \leq 0.01)$. Finally, combination treatment demonstrated a significant elevation of caspase-3 activity level in both cell lines examined. Conclusion: The present data demonstrated targeting miR-27a enhances the anti-tumor effect of SOR in HCC cell lines considering as one of the promising therapeutic targets for advanced HCC management.
\end{abstract}

Keywords: Sorafenib- HCC- miRNAs- miR-27a- HepG2 cells- Huh7 cells

Asian Pac J Cancer Prev, 22 (9), 2951-2958

\section{Introduction}

Hepatocellular carcinoma (HCC) is considered as one of the most commonly diagnosed malignancies and the third-leading cause of cancer-related death in the world after lung and stomach. HCC incidence mainly associated with chronic hepatitis B virus (HBV) and hepatitis $\mathrm{C}$ virus (HCV) infections (Ghouri et al., 2017). HCC has a very low overall 5-year survival rate due to lake of definite and accurate diagnostic tools in its early stages (Golabi et al., 2017). Low survival benefits were recorded for late-stage HCC patients received systemic chemotherapeutic agents (Liu et al., 2015). Sorafenib (Nexavar) is a multikinase inhibitor that targets both RAF and a number of tyrosine kinases, including vascular endothelial growth factor receptors (VEGFRs), and platelet-derived growth factor receptor- $\beta$ (PDGFR- $\beta$ ) (Wilhelm et al., 2005). Despite the fact that sorafenib has been approved in several countries worldwide for advanced HCC patients still yet the drug show unsatisfied clinical outcomes due to its severe side effects as well as drugresistanceleadingto tumor progression and recurrence (van Malenstein et al., 2013). Furthermore, resistance to treatment, tumor recurrence and metastasis shed the light of the importance of providing novel effective therapeutic tactics able to enhance the clinical outcome for HCC patients.

MicroRNAs (miRNAs) are a group of noncoding RNAs that are very conservative with small sequence of nucleotides (17-25nt) which play important regulatory roles in translation of target messenger RNAs through base pairing with the $3^{\prime}$ untranslated region (3'UTR) of their target genes resulting in either degradation or translational inhibition of those genes (Lewis et al., 2005). Growing evidences were accumulated in recent decades emphasizing the crucial role of these small non coding RNAs in regulation of many target genes related to a lot of cellular processes such as cell proliferation, development, differentiation, and apoptosis. Additionally, numerous studies linked between deregulated miRNAs and cancers including $\mathrm{HCC}$ where these miRNAs regulate many 
of the aggressive traits of the disease including tumor progression, metastasis and drug resistance (Zhang et al., 2017; Li et al., 2017).

Interestingly, various studies spotted lights on changes in miRNA expression profiles in many cancer types in response to much therapeutics including sorafenib. It has been shown that miR-26a plays a crucial function for inhibiting angiogenesis and metastasis via targeting VEGFA and HGF. Its low expression was reported in metastatic HCC tissues compared with their corresponding normal referring to its possible therapeutic target against metastasis (Yang et al., 2014). Another study showed an enhancement of sensitivity of HCC cell lines to doxorubicin and vincristine treatment upon upregulating miR-122 expression level through suppressing Bcl-w and cyclin D1 protein levels that are involved (Xu et al., 2011).

miR-27a, one of the miRNA-27 family, was located on chromosome 19. Growing evidence has confirmed its vital role in regulating various biological processes in human cells including proliferation, migration, invasion and metastasis.Furthermore, its clinical significance as a therapeutic agent of cancer and drug sensitivity. It has been demonstrated that miR-27a was significantly overexpressed in different type of tumors including HCC refereeing its oncogenic property (Li et al., 2015). Meanwhile, it behaves as a tumor suppressor in other types of tumor (Bao et al., 2014). Previous study demonstrated that suppression of miR-27a level sensitized colorectal stem cells to TRAIL (Zhang et al., 2017).

Therefore, the aim of the present study was to explore the anti-tumor effect of miR-27a inhibitor in combination with sorafenib treatment on HCC cell lines (HepG2/ Huh7) that might provide a useful strategy for enhancing hepatocellular carcinoma treatment.

\section{Materials and Methods}

\section{Materials}

Dulbecco's modified Eagle's medium (DMEM), fetal bovine serum (FBS), Penicillin and streptomycin were obtained from (Sigma-Aldrich Chemical Co., St. Louis, MO, USA). Sorafenib was purchased from the Bayer Corporation (West Haven, CT). For a $10 \mathrm{mM}$ stock, the $10 \mathrm{mg}$ reconstituted in $1.57 \mathrm{ml}$ DMSO. The final concentration of DMSO in medium was $0.1 \%(\mathrm{v} / \mathrm{v})$.

\section{Cell lines and tissue specimens}

Human hepatocellular carcinoma cell lines HepG2 and Huh7 were purchased from VACSERA (The National Holding Company for Biologics and Vaccines, Cairo, Egypt). The two cell lines were cultured in Dulbecco's Modified Eagle's Medium (DMEM) supplemented with $10 \%$ fetal bovine serum (FBS) and $1 \%$ penicillin/ streptomycin then incubated at $37^{\circ} \mathrm{C}$ in a highly humidified atmosphere incubator with $5 \%(\mathrm{v} / \mathrm{v}) \mathrm{CO}_{2}$ and $95 \%$ air. The cell lines regularly sub-cultured to be in there exponential growth rate.

Nineteen formalin fixed paraffin embedded (FFPE) specimens were obtained from newly diagnosed primary HCC patients at the Egyptian National Cancer Institute, Cairo University. Five FFPE specimens were collected from healthy liver donors' prior transplantation as controls. A written informed consent was obtained from the individual included in the study and the study was approved by the Institutional Review Board of the Egyptian National Cancer Institute, Cairo University.

\section{Cell proliferation assay}

Sulphorhodamine-B (SRB) assay (Sigma-Aldrich Chemical Co., USA) was conducted for assessing the cytotoxic effect of Sorafenib on the growth of HuH-7 and HepG2 cells. Cells were seeded in plate 96-multiwell plate $\left(1 \mathrm{X} 10^{4} \mathrm{cells} /\right.$ well $)$ in fresh medium and cultured for $24 \mathrm{hrs}$ treatment until reaching $50-60 \%$ confluence, then treated with different concentrations of Sorafenib $(3,4,8$, 16 and $32 \mu \mathrm{M}$ ) for $48 \mathrm{hrs}$ to determine the most effective dose to be combined with miR-27ai.Following treatment, cells were fixed with $50 \mu \mathrm{l}$ cold $50 \%$ TCA for $1 \mathrm{~h}$ at $4^{\circ} \mathrm{C}$. Wells were washed once with distilled water and stained for $30 \mathrm{~min}$ at room temperature with $50 \mu 10.4 \%$ SRB dissolved in $1 \%$ acetic acid for $10 \mathrm{~min}$. Unbound SRB was removed by washing five times with $1 \%$ acetic acid. After dried, protein-bound dye was extracted with $100 \mu 1$ of $10 \mathrm{mM}$ Trisbase ( $\mathrm{pH} 10.5)$. Control cells were treated with $0.1 \% \mathrm{DMSO}$ alone and the absorbance was measured at $570 \mathrm{~nm}$ using a microplate reader (Tecan SunriseTM, Germany). Each treatment was done in three independent experiments.

\section{Anti-miR-27a transfection}

HepG2 and Huh7 were incubated at $37^{\circ} \mathrm{C}$ in a $96-$ well plate $\left(1 \mathrm{X} 10^{4}\right.$ cells/well). Just prior to transfection, the media were replaced with $50 \mu \mathrm{l}$ serum free media. MiR-27a inhibitor and miR-NC (purchased from Qiagen, Germany) were transfected into the cells using HiPerFect transfection reagents according to the manufacturer's instruction.After transfection, cells were treated with sub$\mathrm{IC}_{50}$ doses of sorafenib $\left(\mathrm{IC}_{20}\right.$ and $\mathrm{IC}_{35}$ ) for $48 \mathrm{hrs}$. The cells were performed for cell viability, apoptosis, cell cycle, qRT-PCR and caspase-3 assay.

\section{Quantitative RT-PCR}

Total RNA from cell lines and FFPE sections was purified using miRNeasy Mini and miRNeasy FFPE kits (Qiagen, Germany) following manufacture instructions. cDNA was synthesized from 1 ug of RNA using miScript RT II kit (Qiagen) according to the supplied protocol. The expression level of miR-27a was determined using SYBER Green reagent kit (Qiagen). About 5 ng of cDNA was used as template in a $10 \mu \mathrm{lPCR}$ reaction containing 1X SYBR Green master mix, 1X miRNA specific forward primer, and $1 \mathrm{X}$ universal primer. All the RT-qPCR reactions were performed on ViiA7 real-time PCR system (Applied Biosystems, USA). All samples were performed in triplicate. U6 snRNA was used as an endogenous control for normalization. Data were expressed as fold change, using the $2^{-\Delta \Lambda C t}$ method.

\section{Enzyme-linked immune sorbent assay for Caspase-3}

The concentrations of Caspase-3 level was determined on cell lysate of transfected/untransfected followed with sorafenib treated cellsfor $48 \mathrm{hrs}$ comparing to negative 
control using ELISA kit (BioSource, CA, USA) according to the manufacturer's instructions. All samples were assayed in triplicate and standard curve was plotted and used to calculate the concentrations.

\section{Cell cycle assay}

After $48 \mathrm{hrs}$ treatment with sorafenib, the transfected/ untransfected cells and negative control were harvested and washed twice with cold PBS. The cell suspension was adjusted to a concentration of 1 X $10^{6}$ cells per $\mathrm{ml}$. Cells were fixed in $100 \%$ ethanol at $4^{\circ} \mathrm{C}$ overnight then cells were resuspended in $200 \mu \mathrm{L} 1 \mathrm{X}$ propidium iodide (PI), incubated at $37^{\circ} \mathrm{C}$ in the dark for $20-30$ min placed on ice. G0 /G1, S and G2/M phases' populations were detected by PI detector with flow cytometry (Beckman Coulter, USA) and analyzed by flowing software version 2.5.1(Turka centre of biotechnology, Turka uni, Finland).

\section{Cell apoptosis analysis}

Annexin V-FITC/PI (fluorescein isothiocyanate/ propidium iodide) apoptosis detection kit (BestBio, Shanghai, People's Republic of China) was used according to the manufacturer's protocols for detection of apoptotic cells via flow cytometry in transfected and untransfected cells before and after sorafenib treatment comparing to negative control. Briefly, cells were collected and centrifuged for $10 \mathrm{~min}$ at 1,200 rpm. After washing with PBS, cell suspension was adjusted to a concentration of $1 \mathrm{X} 10^{6}$ cells per $\mathrm{ml}$ and stained with $10 \mu \mathrm{L}$ of Annexin V-FITC for 15 minutes and $5 \mu \mathrm{L}$ of PI for 5 minutes at $4^{\circ} \mathrm{C}$ in the dark. Cells were then analyzed using FITC signal detector and PI detector with flow cytometry (Beckman Coulter, USA), and flowing software version 2.5.1 (Turka centre of biotechnology, Turka uni, Finland)

\section{Statistical analysis}

Statistical analyses were performed using GraphPad Prism version 7 for windows (GraphPad Software, CA,
USA).Data are presented as mean \pm standard deviation (SD). Multiple comparisons were carried out using one way analysis of variance (ANOVA) followed by Tukey test for post-hoc .Statistical significance was acceptable at a level of $\mathrm{p}$-value $<0.05$. The two-tailed Student's t test was used for all real time RT-PCR, cell cycle, apoptosis and caspase- 3 level data. P-value threshold $<0.05$ was considered significant.

\section{Results}

\section{Effect of sorafenib on cell proliferation}

Both cell lines used in the present study (HepG2/Huh7) demonstrated slightly difference in their sensitivity to sorafenib. HepG2 cells exhibited more sensitivity rather than Huh7 cells after treatment for $48 \mathrm{hrs}$. The calculated IC50 for HepG2 and Huh7 cells was $8 \mathrm{uM}$ and $10 \mathrm{uM}$ respectively. Based on this data, sub lethal doses of sorafenib $\left(\mathrm{IC}_{20}\right.$ and $\left.\mathrm{IC}_{35}\right)$ were selected for treatment of cells in the subsequent experiments.

\section{Effect of combinedtreatment of miR-27a inhibitor/ Sorafenib on proliferation rate of HepG2 and Huh7 cell lines}

Combining treatment of Anti-miR-27a and sorafenib was performed to determine their anti-tumor effect on HepG2 or Huh7 cells proliferation. A significant decreasing in proliferation was reported in both cell lines (HepG2 and Huh7) transfected with miR-27a inhibitor alone by $37 \%$ and $18 \%$ (Figure 1) compared with NTC (p $<0.05)$. While, combined treatment of miR-27a inhibitor with sorafenib $\left(\mathrm{IC}_{20}\right)$ resulted in a significant inhibition in cell viability by $40 \%$ and $71 \%$ in HepG 2 cells and Huh7 respectively compared to NTC $(\mathrm{p}<0.05)$. The combined treatment of miR-27a inhibitor with higher dose of sorafenib $\left(\mathrm{IC}_{35}\right)$ resulted in a higher significant inhibition in cell viability by $52.5 \%$ and $76.5 \%$ in Hepg2 cells and Huh7 respectively compared to NTC $(p<0.05)$.

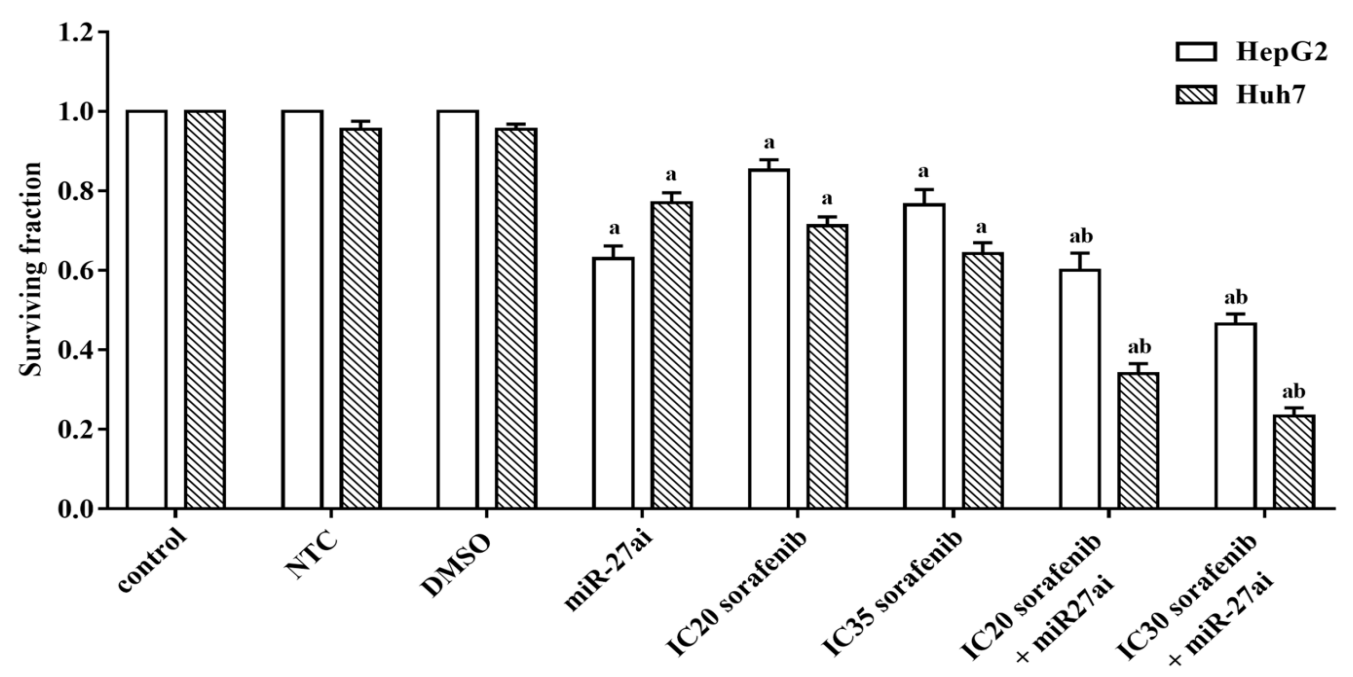

Figure 1. Effect of different doses of sorafenib $\left(\mathrm{IC}_{20}\right.$ and $\left.\mathrm{IC}_{35}\right)$ on the surviving fraction of HepG2 and Huh7 cells transfected with $50 \mathrm{nM}$ miR-27a inhibitor for $48 \mathrm{hrs}$. The results are expressed as the mean $\pm \mathrm{SD}$ of 3 independent experiments. The statistical significance of the results was analyzed using one way ANOVA followed by Tukey multiple comparison test. a Significantly different from controls (control, NTC and DMSO), b from sorafenib only treated cells $(\mathrm{p} \leq 0.05)$. 


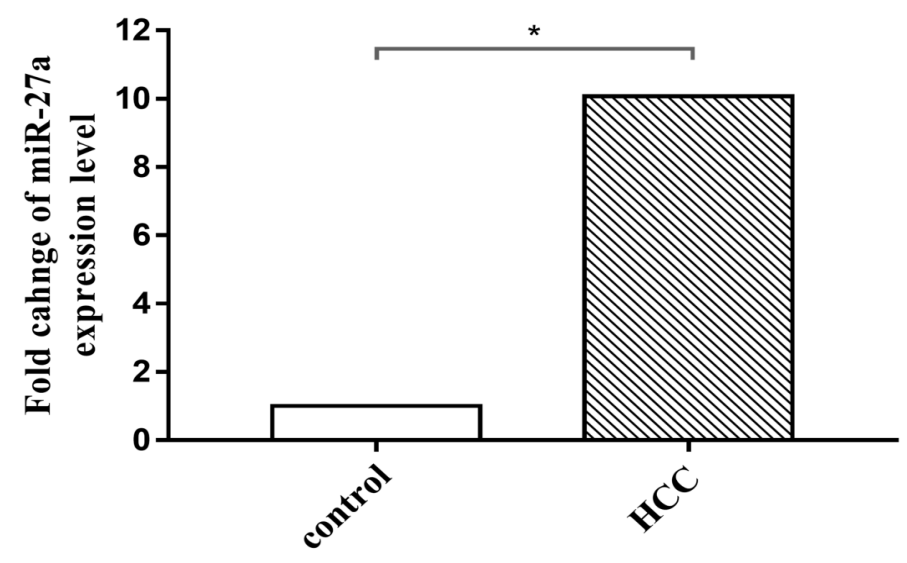

Figure 2. Fold Changes of miRNA-27a Expression Level Using qRT-PCR Assay in RNA Isolated from FFPE Tissue Sample of Primary Hepatocellular Carcinoma Patients Compared with Control Tissues from Healthy Volunteers. The results are expressed as the mean $\pm \mathrm{SD}$ of 3 independent experiments. The statistical significance of the results was analyzed using the two-tailed Student's t test $(* \mathrm{p} \leq 0.05)$.

Negative control and solvent (DMSO) did not show any significantly effect on cell viability for both cell lines. The data revealed that combining the antisense miR-27a with sorafenib could enhance the antitumor effect of sorafenib through declining the number of viable cells in both cell lines. Accordingly, we selected the best anti-proliferative effect of sorafenib $\left(\mathrm{IC}_{35}\right)$ with miR-27ai for the analysis of cell cycle, apoptosis and caspase- 3 assays.

Effects of Sorafenib or miR-27a inhibitor on Expression level of miR-27a in HepG2 and Huh7 cell lines

Using quantitative RT-PCR assay, a significant up-regulation of miR-27a expression was reported in RNA isolated from FFPE specimens of 19 primary HCC patients analyzed compared with their corresponding healthy controls (Figure 2). The mean fold change recorded was about $10.072(\mathrm{P}<0.0206)$. This data confirmed overexpression of miR-27a in primary HCC tissues that refer to its oncogenic function.

Moreover, we investigated whether sorafenib or miR$27 \mathrm{a}$ inhibitor influence the expression level of miR-27a in HepG2 and Huh-7 cell lines. As shown in Figure 3, a significant decrease in miR-27a expressionlevel was reported for both cell lines after treatment with sorafenib where $\log$ fold changes of miR-27a level were- 0.5 and -1.5 for both cell lines respectively $(\mathrm{P}<0.01)$.Furthermore, highly significant down-regulated ( -3.2 and -2.8$)$ miRNA-27a expression was detected in both cell lines after transfection with miR-27ai indicating its successful transfection process $(\mathrm{P}<0.01)$.

Effect of combined treatment (miR-27ai and sorafenib) on cell cycle and apoptosis of HepG2 and Huh7 cell lines

We investigated the effect of combination of anti-miR-27a and sorafenib $\left(\mathrm{IC}_{35}\right)$ on cell cycle progression using flow cytometry for HepG2 and Huh7cell lines. A significant decreasing in the number of cells in S-phase was reported when cells treated with miR-27ai or sorafenib alone for both cell lines $(p<0.05)$. Furthermore, a significant decreasing was recorded when cells exposed to combined treatment (miR-27ai+sorafenib).A significant increasing was also reported in number of cells in sub G0-phase for combined treated cells $(\mathrm{p}<0.05)$ compared with control untreated cells (Figure 4).

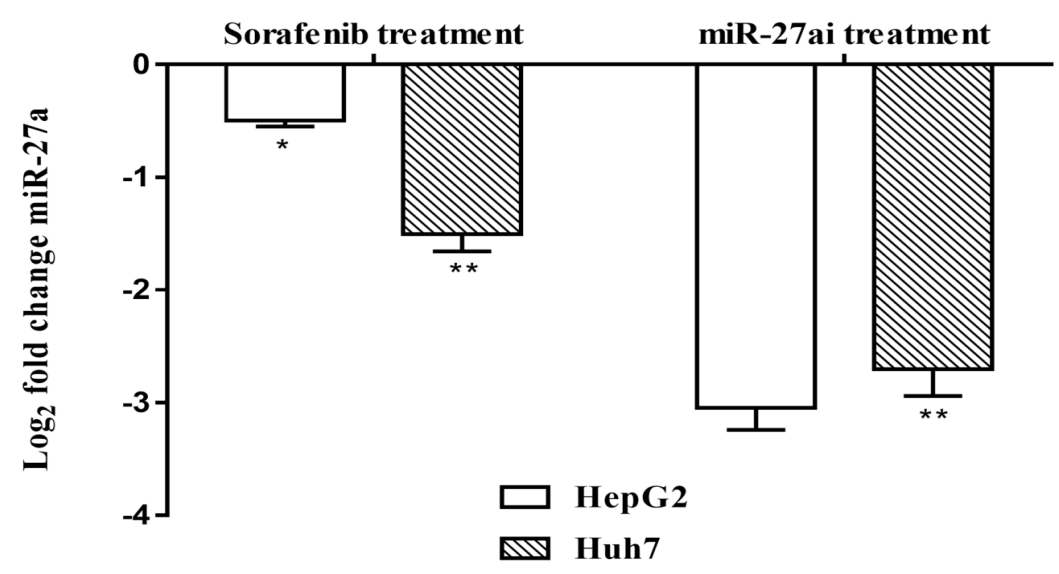

Figure 3. Gene Expression Analysis of miR-27a Using qRT-PCR Assay in Hepatocellular Carcinoma Cells, HepG2 and Huh7, after Treatment with Sorafenib or miR-27a Inhibitor Compared with Control Untreated Cells. Data were represented as mean values \pm SD for three independent experiments. The statistical significance of the results was analyzed using the two-tailed Student's t test $(* \mathrm{p} \leq 0.05, * * \mathrm{p} \leq 0.01)$. 


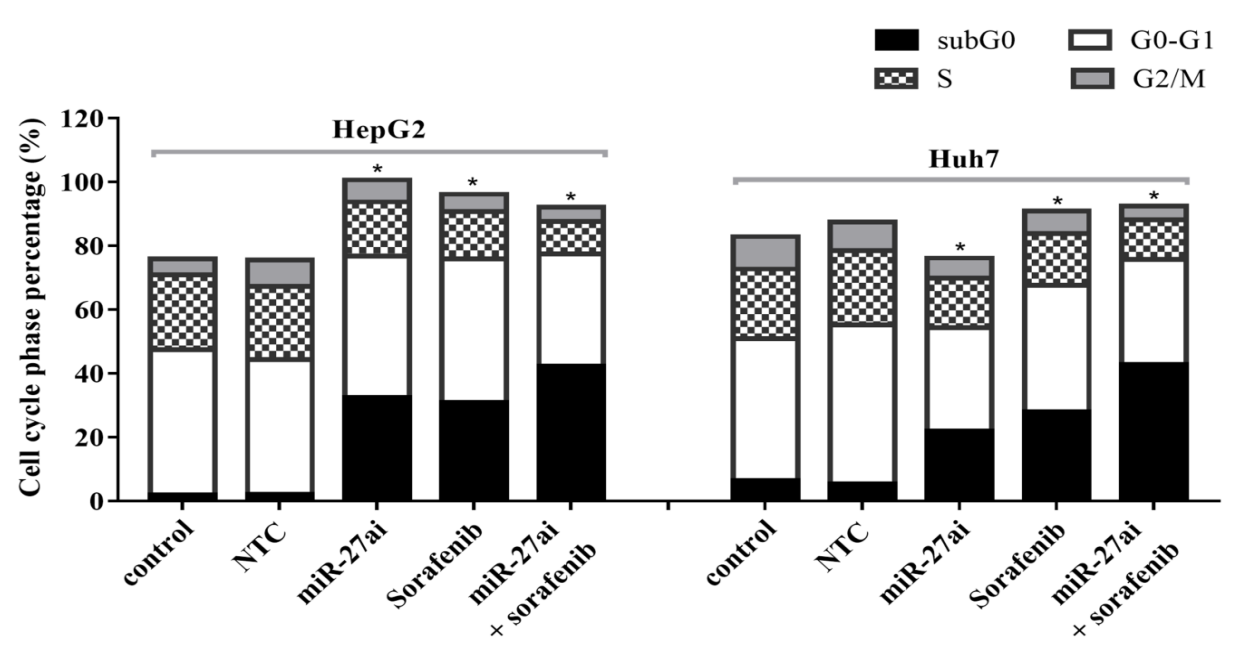

Figure 4. Flow Cytometry of Cell Cycle Analysis in HepG2 and Huh7 Cell Lines Using PI Staining. The percentage of each cell cycle for both cells lines in different treated groups was represented. The statistical significance of the results was analyzed using the two-tailed Student's t test $(* \mathrm{p} \leq 0.05)$.

In this study we next evaluated the apoptotic induction in HepG2 and Huh7 cells which co-treated with anti-miR-27a followed by sorafenib $\left(\mathrm{IC}_{35}\right)$ via assessment the percentage of apoptotic cells by flow cytometry. Significant increase in apoptotic cell populations in HepG2 and Huh7 cells was observed when treated with sorafenib alone $(\mathrm{p}<0.05)$ and this rate demonstrated highly significant for cells transfected with miR-27a inhibitor followed by treatment with sorafenib compared with control cells $(p<0.01)$. The percentage of apoptotic rates recorded for HepG2 transfected with miR-27ai, sorafenib alone, and combined treatment were about $6.15 \%, 23.1 \%$ and 65.9 versus control $1.21 \%$ respectively.Furthermore, the percentage of apoptotic rates for Huh7 cells recorded were $0.87 \%, 33.3 \%$ and $70.1 \%$ versus control $0.32 \%$ respectively as indicated in Figure 5.

To further verify the anti-tumor effect of miR-27ai+ sorafenib induced cell apoptosis, we examinedthe level of caspase- 3 activity, a hallmark of apoptosis, on both cell lines using Elisa assay. As shown in figure 6, there is a significant increasing in its level for miR-27ai transfected cells or sorafenib treated cells alone which became highly elevated for combination treated cells compared with either of control or single agent treatment $(p<0.05)$.

\section{Discussion}

Therapeutic modulation of a single miRNA may affect many pathways simultaneously to achieve more clinical benefit (Ebert et al., 2007). Hence targeting aberrantly expressed miRNA is logical and attractive. The widespread overexpression of miR-27a in cancer has led to the belief that miR-27a is an oncogenic microRNA. Cell culture and animal experiments supported this speculation, showing that the down-regulation of miR-27a expression can suppress cell proliferation and slow tumor growth. Some reports demonstrated the oncogenic role of miR-27a which plays important role in the development of HCC

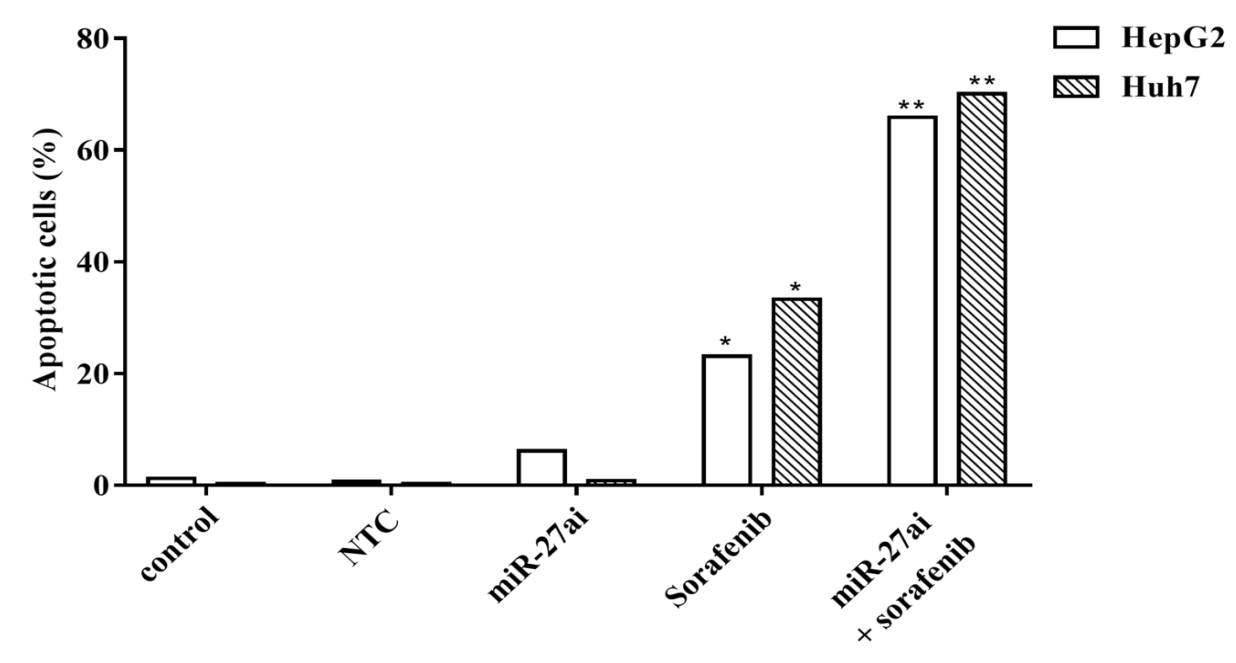

Figure 5. Flow Cytometry of Apoptosis Analysis in Hepg2 and Huh7 Annexin V-FITC and PI Staining Assay. Percentage of apoptosis was represented for both cells lines in different group analyzed. The statistical significance of the results was analyzed using the two-tailed Student's t test $(* \mathrm{p} \leq 0.05)$. 


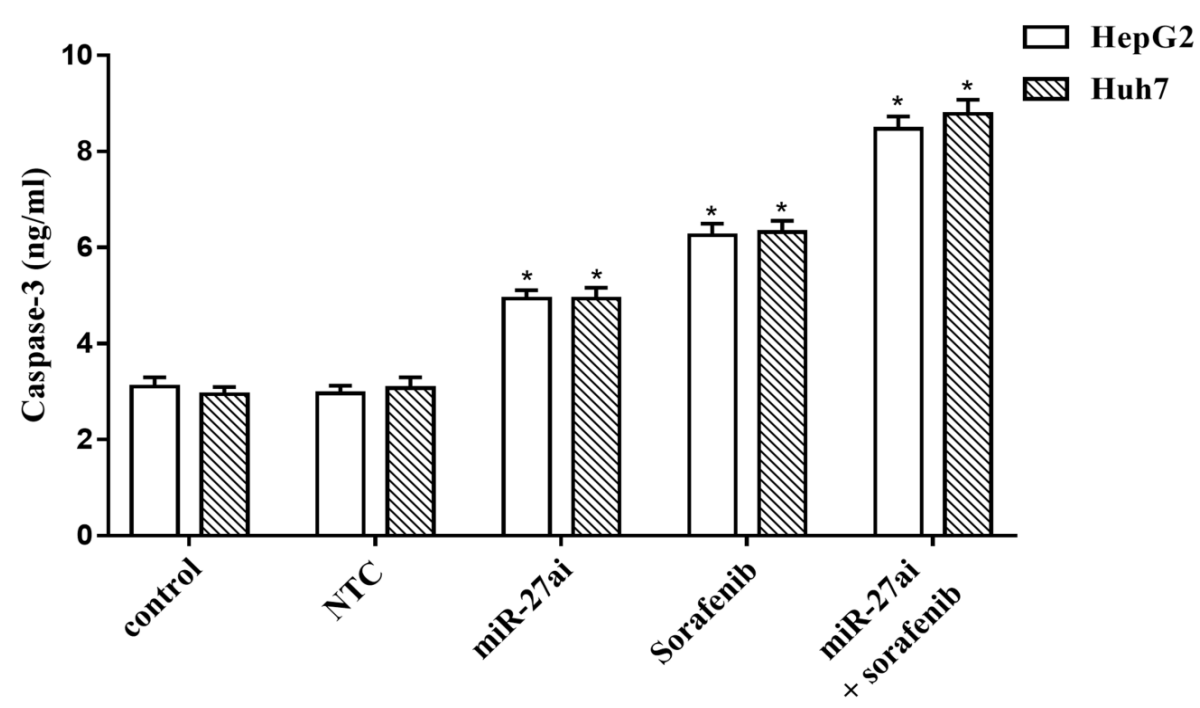

Figure 6. Caspase-3 Level (ng/ml) Hepatocellular Carcinoma Cells, HepG2 and Huh7, after Treatment with Sorafenib, miR-27a Inhibitor and Combination Compared with Control Untreated Cells Using Elisa Assay. Data were represented as mean values \pm SD for three independent experiments. The statistical significance of the results was analyzed using one way ANOVA followed by Tukey multiple comparison test. a Significantly different from controls (NC, NTC and solvent), $b$ from sorafenib only treated cells $(\mathrm{p} \leq 0.05)$ considering significant

and might thus be a new therapeutic and prognostic target in HCC patients (Li et al., 2015).

Several recent studies confirmed the protooncogenic characteristic of miR-27a in different types of cancer. It has been shown that overexpression of miR-27a was associated with high cell proliferation and metastasis in gastric cancer cells through mediating suppression of $\mathrm{PH}$ domain and leucine-rich repeat protein phosphatase 2 (PHLPP2) leading to stimulation of the AKT/GSK3B pathway (Ding et al., 2017). Moreover, overexpression of miR-27a enhanced tumor metastasis by stimulating epithelial-mesenchymal transition process in breast cancer which added further evidences for its potential application in cancer therapy (Jiang et al., 2018). High expression of miR-27a level detected intissues ofprimary HCC patients in the current data are consistant with that previously reported studies confirming its oncogeneic role in HCC.

The discovery of targeted therapy with multi-tyrosine kinase inhibitors, such as sorafenib has set abreakthrough for patients with advanced cancer. Its antitumor mechanisminvolving the inhibiton mostly known pathways associated with hepatocarcinogenesis include the Ras/Raf/ MAP/ERK, the PI3K/Akt/mTOR, the Wnt/ $\beta$-catenin and the JAK/STAT pathways (Wilhelm et al., 2005) but its mechanism of action still has not been fully elucidated. Emmerging evidences considering miRNAs as important key players in HCC pathogenesis. Numerous experimental and clinical studies revealed that the aberrant expression of miRNAs is associated with the progression of hepatocellular carcinoma (Callegari et al., 2013; Lu et al., 2005). Moreover, various studies highlighted changes in miRNA expression profiles in response to sorafenib and other therapeutics (Peveling-Oberhag et al., 2015; Stiuso et al., 2015). A study done on HCC cells demonstrated that miR-142-3p sensitised cells to sorafenib by promoting apoptosis and inhibiting cell growth and proliferation via targeting autophagy related 5 (ATG5) and autophagy- related 16-like 1 (ATG16L1) which are the main targets of autophagy regulating miR-142-3p (Zhang et al., 2018). Delcining of miR-221 overexpression level using its inhibitor modulate sensitized HCC cells via induction of caspase-3, a pro-apoptotic target of miR-221 (Fornari et al., 2017). It has been indicated recently that miR-140-3p can enhance the antitumor effect of sorafenib via targeting pregnane X recptor (PXR) resulting down-regulation of drug-resistance related genes in HCC cells (Li et al., 2018).

We explored the feasibility of regulation of miR-27a using chemically modified anti-miR Oligos alone and in combination of Sorafenib in HCC cells. Our data showed that combining of antisense miR-27a Oligos with sorafenib sensitized HepG2 and Huh7 to sorafenibinduced cell death compared to sorafenib-onlytreated cells and controls, indicating that antisense miR-27acould potenitiate the antitumor effect of sorafenib through declining the number of viable $\mathrm{HCC}$ cells in vitro. Previous study demonstrated using a lentiviral vector to stably expression of anit-miR-27a targeting the oncogenic miRNA declines proliferation of glioma cell line suggesting its potential role for suppression of malignancy (Feng et al., 2012). Xu et al., (2013) proposed that inhibtion of miR-27a by its antisense oligoenhanced the response of ovarian cancer cell line SKOV3 to Genistein and decrease cell growth and metastasiss by upregulating the Sprouty2, the putative miR-27a target gene.

An up-regulation of miR-27a was observed significantly in HCC tissues and different cell lines. Furthermore, its down-regulation using miR-27a inhibitor declined proliferation, induced apoptosis and blocked the G1/S cell cylce transition via targeting the 3'-untranslated region of peroxisome proliferator-activated receptor $\gamma$ (PPAR $\gamma$ ) using dual luciferase assay (Li et al., 2015). In gastric cancer cells, the reduction of miR-27a inhibited cell growth in both in vitro and nude mice assays which referred to its important role in cell proliferation (Zhao 
et al., 2011). Moreover, the most exciting finding in the present study was observed where a significantdeclining of cell count in S-phase of combined treated cells compared with either sorafenib or miR-27a inhibitor alone as shown by cell cycle analysis. Previous study done on the same cell lines reported reduction of cell counts in $\mathrm{S}$ phase upon treatment with anti-miR-27a olignonucleotides ( $\mathrm{Li}$ et al., 2015)

Apoptosis is the one of dysfunctioning signaling pathways in HCC. The effects of administration of sorafenib on inducing apoptosis were investigated in many studies (Garten et al., 2019; Bahman et al., 2018).

In the current work, apoptosisanalysis conducted by flow cytometry demonstrated a sigificant increase in apoptotic rate in cells treated with combination therapy compared with cells treated with either sorafenib or miR-27a inhibitor alone. Finding of Li et al., (2018) demonstrated a significant increase in apoptotic rate in HepG2 transfected with miR-27a inhibitor whichverifiedthe additionnal effect of combinational therapy (antisense miR-27a and sorafenib) on apoptotic rate of hepatocellular carcinoma cells.

To gain insight the molecular mechanisms in miR-27a mediated apoptosis ,caspase-3 level was assesed in HepG2and Huh7 transfected cells withantisense miR-27a. The results showed a significant increase in the caspase- 3 in both transfected cells compared to controls. Remarkably trasfected cells which co-treated with sorafenib demonstrated an enhancement in caspase-3 level compared to treatmet with either sorafenib or miR-27a alone. The elevated level of the assessed caspase- 3 upon transient ransfection of miR-27a inhibitorin HepG2 and Huh7 might proposed to the negative correlation between miR-27a and APAF-1, a key mediator in cytochrome C-dependent apoptotic pathway (Zhang et al., 2017). The author considered APAF-1 is the direct target of miR-27a.

Our finding data demonstrated that blocking miR-27a plays a crucial role in cell proliferation, cell cylce and apoptosis process in hepatocellular carcinoma cell lines. Furthermore, the antitumor effect of miR-27a inhibitor in combination with sorafenib on hepatocellular cell lines might alter its therapeutic strategy.

\section{Acknowledgements}

We would like to thank all the members of Abdel Wahab A.A. laboratory.

\section{Author Contribution Statement}

AA, conceived, designed and supervised the study. EA, performed the experiments and analyzed the data. AA, shared in study design and technical guidance of study. $\mathrm{AA}, \mathrm{AA}, \mathrm{MA}$, and HS, analyzing and interpretation of data as well as drafting the manuscript. All authors read and approved the final manuscript.

\section{References}

Bahman A, Abaza M, Khoushiash S, Al-attiyah R (2018). Sequence dependent effect of sorafenib in combination with natural phenolic compounds on hepatic cancer cells and the possible mechanism of action. Int JMol Med, 42, 1695-1715.

Bao Y, Chen Z, Guo Y, et al (2014). Tumor suppressor microRNA-27a in colorectal carcinogenesis and progression by targeting SGPP1 and Smad2. PLoS One, 9, e105991.

Callegari E, Elamin BK, Sabbioni S, Gramantieri L, Negrini M (2013). Role of microRNAs in hepatocellular carcinoma: a clinical perspective. Onco Targets Ther, 6, 1167-78.

Ding L, Zhang S, Xu M, et al (2017). MicroRNA-27 a contributes to the malignant behavior of gastric cancer cells by directly targeting $\mathrm{PH}$ domain and leucine-rich repeat protein phosphatase 2. J Exp Clin Cancer Res, 36, 45.

Ebert MS, Neilson JR, Sharp PA (2007). MicroRNA sponges: competitive inhibitors of small RNAs in mammalian cells. Nat Methods, 4, 721-6.

Feng SY, Dong CG, Wu WK, et al (2012). Lentiviral expression of anti-microRNAs targeting miR-27a inhibits proliferation and invasiveness of U87 glioma cells. Mol Med Rep, 6, 275-81.

Fornari F, Pollutri D, Patrizi C, et al (2017). In hepatocellular carcinoma miR-221 modulates sorafenib resistance through inhibition of caspase-3-mediated apoptosis. Clin Cancer Res, 23, 3953-65.

Garten A, Grohmann T, Kluckova K, et al (2019). SorafenibInduced Apoptosis in Hepatocellular Carcinoma Is Reversed by SIRT1. Int J Mol Sci, 20, 4048.

Ghouri YA, Mian I, Rowe JH (2017). Review of hepatocellular carcinoma: Epidemiology, etiology, and carcinogenesis. $J$ Carcinog, 16, 1.

Golabi P, Fazel S, Otgonsuren M, et al (2017). Mortality assessment of patients with hepatocellular carcinoma according to underlying disease and treatment modalities. Medicine (Baltimore), 96, e5904.

Jiang G, Shi W, Fang H, Zhang X (2018). miR 27a promotes human breast cancer cell migration by inducing EMT in a FBXW7 dependent manner. Mol Med Rep, 18, 5417-26.

Lewis BP, Burge CB, Bartel DP (2005). Conserved seed pairing, often flanked by adenosines, indicates that thousands of human genes are microRNA targets. Cell, 120, 15-20.

Li C, Miao R, Liu S, et al (2017). Down-regulation of miR$146 \mathrm{~b}-5 \mathrm{p}$ by long noncoding RNA MALAT1 in hepatocellular carcinoma promotes cancer growth and metastasis. Oncotarget, 8, 28683-95.

Li J, Zhao J, Wang H, et al (2018). MicroRNA-140-3p enhances the sensitivity of hepatocellular carcinoma cells to sorafenib by targeting pregnenolone $\mathrm{X}$ receptor. Onco Targets Ther, 11, 5885-94.

Li S, Li J, Fei BY, et al (2015). Mir-27a promotes hepatocellular carcinoma cell proliferation through suppression of its target gene peroxisome proliferator-activated receptor gamma. Chin Med J (Engl), 128, 941-7.

Liu CY, Chen KF, Chen PJ (2015). Treatment of Liver Cancer. Cold Spring Harb Perspect Med, 5, a021535.

Lu J, Getz G, Miska EA, et al (2005). MicroRNA expression profiles classify human cancers. Nature, $\mathbf{4 3 5}$, 834-838.

Peveling-Oberhag J, Döring C, Hartmann S, et al (2015). Feasibility of global miRNA analysis from fine-needle biopsy FFPE material in patients with hepatocellular carcinoma treated with sorafenib. Clin Sci, 128, 29-37.

Stiuso P, Potenza N, Lombardi A, et al (2015). MicroRNA-423$5 p$ promotes autophagy in cancer cells and is increased in serum from hepatocarcinoma patients treated with sorafenib. Mol Ther Nucleic Acids, 4, e233.

van Malenstein H, Dekervel J, Verslype C, et al (2013). Long-term exposure to sorafenib of liver cancer cells induces resistance with epithelial-to-mesenchymal transition, increased invasion and risk of rebound growth. Cancer 
Lett, 329, 74-83.

Wilhelm SM, Carter C, Tang L, et al (2005). BAY 43-9006 exhibits broad spectrum oral antitumor activity and targets the RAF/MEK/ERK pathway and receptor tyrosine kinases involved in tumor progression and angiogenesis. Cancer Res, 64, 7099-7109.

Xu L, Xiang J, Shen J, et al (2013). Oncogenic MicroRNA-27a is a target for genistein in ovarian cancer cells. Curr Med Chem Anticancer Agents, 13, 1126-32.

Xu Y, Xia F, Ma L, et al (2011). MicroRNA-122 sensitizes HCC cancer cells to adriamycin and vincristine through modulating expression of MDR and inducing cell cycle arrest. Cancer Lett, 310, 160-9.

Yang X, Zhang XF, Lu X, et al (2014). MicroRNA-26a suppresses angiogenesis in human hepatocellular carcinoma by targeting hepatocyte growth factor cMet pathway. Hepatology, 59, 1874-85.

Zhang K, Chen, J, Zhou H, et al (2018). microRNA-142-3p targets ATG5/ATG16L1 to inactivate autophagy and sensitize hepatocellular carcinoma cells to sorafenib. Cell Death Dis, 9, 312.

Zhang LL, Guo YJ, Zhao CN, Gao JY (2015). Effects and mechanism of miR-214 on hepatocellular carcinoma. Asian Pac J Trop Med, 8, 392-8.

Zhang R, Xu J, Zhao J, Bai J (2017). Knockdown of miR 27 a sensitizes colorectal cancer stem cells to TRAIL by promoting the formation of Apaf 1 caspase 9 complex. Oncotarget, 8, 45213-23.

Zhao X, Yang L, Hu J (2011). Down-regulation of miR-27a might inhibit proliferation and drug resistance of gastric cancer cells. J Exp Clin Cancer Res, 30, 55.

This work is licensed under a Creative Commons AttributionNon Commercial 4.0 International License. 